Aus: "In ihrer rechten Hand hielt sie ein silbernes Messer mit Glöckchen ...": Studien zur indischen Kultur und Literatur, hrsg. von Anna Aurelia Esposito u.a. - Wiesbaden:

Harrassowitz Verlag. - 2015, S. 337-344

\title{
Pretas und arme Seelen, Ein Beispiel für eine parallele Entwicklung religiöser Inhalte und Strukturen in Europa und Asien
}

\author{
Sebastian Stinzing
}

Im Petavatthu, einem buddhistischen Text der Theravāda-Schule ${ }^{1}$ aus den ersten Jahrhunderten nach der Zeitenwende, wurden Geschichten über das Schicksal von Verstorbenen gesammelt, die wegen eines schlechten Lebenswandels nach ihrem Tod als Petas ${ }^{2}$ wiedergeboren werden. In einer Geschichte geht es um Nandā, eine Frau, die sich zu Lebzeiten ihrem Mann gegenüber unziemlich benommen hat. Sie erlangt eine Wiedergeburt als Totengeist und begegnet in dieser Gestalt ihrem trauernden Ehemann, der sie aber kaum wiedererkennt:

\section{Das Lied von der Nandā (Nandāpetavatthu, Pv II, 4)}

1. Schwarz und von gräßlichem Aussehen bist du, rauh am Körper, schrecklich anzusehen, mit rötlichen Augen und gelblichen Zähnen, ich glaube nicht, da $\beta$ du ein Mensch bist.

2. Ich bin Nandā, o Nandasena, früher war ich deine Gattin, da ich sündige Tat getan, bin ich hier in die Welt der Petas gekommen.

3. Was für Böses hast du getan?

4. Ich war jähzornig und rau, ohne Ehrfurcht gegen dich; dafür, da $B$ ich Böses gesagt habe, bin ich hier in die Welt der Petas gekommen.

5. Nimm hier, ich gebe dir das Obergewand, Dieses Kleid zieh an, und wenn du es angezogen hast, so komm, ich will dich nach Hause führen.

6. Kleidung, Speise und Trank wirst du erhalten, wenn du nach Hause gegangen, und deine Söhne wirst du sehen und die Schwiegertöchter.

7. Das von deiner in meine Hand gegebene kommt nicht mir zugute,

1 Das Alter des Petavatthu ist schwer zu bestimmen. In seiner jetzigen Form ist es ein relativ ,junger" Text, der sich in seiner abgeschlossenen Form spätestens auf das fünfte nachchristliche Jahrhundert datieren lässt. Zu diesem Zeitpunkt wurde der Kommentar des Dhammapāla zu dem Text niedergeschrieben.

2 Buddhistische Hungergeister. 
Bhikkus, mit Tugend begabt, welche die Leidenschaft abgelegt, und in der Schrift bewandert:

8. Die erquicke mit Speise und Trank, und übertrage die Gabe auf mich, dann werde ich glücklich sein und über alle Wonne verfügen.

9. Er versprachs ihr, verteilte reichlich Gaben, Speise, Trank und Kuchen, Kleider, Lager und Sitze, Schirm, Salbe und Blumen, und mancherlei Sandalen.

Bhikkus, mit Tugend begabt ohne Leidenschaft, in der Schrift bewandert, mit Speise und Trank erfrischend, übertrug er auf sie die Gabe. ${ }^{3}$

Im Dialogus Miraculorum ${ }^{4}$, einem lateinischen Text des 13. Jh., findet sich eine ähnliche Geschichte, in der ein verstorbener Bayer seiner Witwe erscheint:

\section{Wie ein Bayer seiner Gattin nach dem Tode erschien \\ (De Bauwaro qui post mortem uxori apparens, dixit sibi eleemosynas nihil profuisse. Dialogus Miraculorum, 12. XIX)}

Nicht viele Jahre sind verstrichen, seit ein sehr reicher Dienstmann des Herzogs von Bayern starb. Eines Nachts ward die Burg, worin seine Gattin schlief, dermaßen erschüttert, daß man glaubte, es sei ein Erdbeben. Und siehe, die Tür der Kammer, worin jene lag, sprang auf, und geführt von einem pechschwarzen Riesen, der ihn an den Schultern gepackt hielt, trat ihr Gatte herein. Da sie diesen sah und erkannte, ließ sie ihn auf dem Sitz ihres Bettes sitzen. Sie erschrak nicht im mindesten, und weil es kalt war und sie nur mit einem Hemde bekleidet war, so legte sie ihm einen Teil ihrer Decke über die Schultern. Als sie ihn nach seinem Zustand befragte, antwortete er traurig: Zu ewiger Qual bin ich verdammt. Über dieses Wort sehr erschrocken, sagte sie: Was sprecht Ihr? Habt Ihr nicht große Almosen gegeben? Stand nicht eure Pforte allen Pilgern offen? Nützen Euch diese Guttaten nichts? Nichts nützen sie mir im ewigen Leben, antwortete er, weil ich sie aus eitler Ruhmsucht und nicht aus Nächstenliebe getan habe. Als sie ihn nach anderen Dingen befragen wollte, sagte er: Mir ist gestattet worden, dir zu erscheinen, aber ich darf hier nicht verweilen. Sieh, mein Führer, der Teufel, steht draußen und erwartet mich. Wenn aber alle Blätter auf den Bäumen in Zungen verwandelt würden, sie könnten meine Qualen nicht ausdrücken. Hierauf wird er herausgeholt und weggetrieben; bei seinem Abgang wird die ganze Burg wie vorher erschüttert, und noch lange hört man sein Jammergeschrei. Diese Erscheinung war sehr bekannt in Bayern und ist es noch heute, wie es bezeugt unser Mönch Gerhard, einst Domherr in Regensburg, der sie uns erzählt hat. ${ }^{5}$

Die beiden Texte, Petavatthu und Dialogus Miraculorum, sind das Produkt unterschiedlicher Kulturen, die nicht nur räumlich und zeitlich getrennt sind, sondern sich in gesellschaftlicher Organisation, Religion und literarischem Hintergrund beträchtlich unterscheiden. Trotzdem haben die beiden Literaturtraditionen Formen

3 Die Übersetzung folgt Stede 1914: 82-83.

4 Im Dialogus Miraculorum hat der Mönch Caesarius von Heisterbach eine Reihe von wunderbaren Begebenheiten gesammelt und in die Rahmenhandlung eines Dialoges zwischen einem Novizenmeister und Schüler eingebettet.

5 Die Übersetzung folgt Herles 1990: 285-6. 
von Gespenstergeschichten hervorgebracht, die sich in ihrer Struktur stark gleichen. Die Beispiele sind dabei keine alleinstehenden Sonderfälle, sondern die beiden Textsammlungen sind voll von strukturell ähnlichen Erzählungen.

Wie lässt sich diese Ähnlichkeit erklären? Eine Übertragung des Grundmodells der Erzählung durch Diffusion kann ausgeschlossen werden, die kulturellen Kontakte zwischen Indien und Europa waren nicht stark und unmittelbar genug, um so etwas zu leisten. Eine vorgeschichtliche Überlieferung des Motivs, das sich dann über so lange Zeit unverändert erhalten hat, ist auch nicht wahrscheinlich.

Mehrere Anhaltspunkte sprechen für eine dritte Alternative, nämlich eine parallele Entwicklung des Geisterkonzeptes in den beiden Kulturkreisen.

\section{Evolutionäre Anlagen und „supernatural agency“}

Grundlagen für den Gespensterglauben in Aufbau und Funktion des menschlichen Gehirns

Fast jede Kultur kennt die Vorstellung von Totengeistern. ${ }^{6}$ Die grundsätzliche Konzeption solcher Totengeister ist sehr ähnlich, die spezielle Ausprägung aber immer stark von der jeweiligen Kultur abhängig. Erkenntnisse aus der Evolutionspsychologie sprechen dafür, dass das Vorhandensein dieser Vorstellung auf Eigenheiten im Aufbau des Gehirns zurückgeht, die sich im Lauf der menschlichen Evolution herausgebildet haben.

Ein aktuelles Modell der Gehirnfunktionen geht vom Zusammenspiel verschiedener „Module“ und Inferenzsysteme. Diese erlauben es, aus den anfallenden Sinnesdaten der Umgebung und vorheriger Erlebnisse Schlüsse für das eigene Handeln zu ziehen. Scott Atran, ein Anthropologe, der sich mit den evolutionären Grundlagen religiösen Denkens beschäftigt hat, schreibt dazu:

"A naturally selected, mental module is functionally specialized to process, as input, a specific domain of recurrent stimuli in the world that was particularly relevant to hominid survival. The module spontaneously produces, as output, groupings of stimuli into categories as well as inferences about the conceptual relationships between these categories. The innately constrained cognitive structure of this output presumably was designed under natural selection. It allowed humans to adaptively navigate ancestral environments by responding rapidly and economically to important, statistically repetitive tasks such as distinguishing predator from prey and friend from foe."

Eines dieser Module dient der Erkennung intelligenter ,agents“. Für den Menschen ist dieses Modul, das auch die Empathie und das Verstehen von Motivationen anderer erlaubt, eines der wichtigsten. In den Worten Atrans:

Agents are entities that instigate and control their own actions as well as those of other objects and agents. Developmental and cognitive psychology have experimented with several related theories of how humans come to make sense of one another. An overarching theoretical framework has emerged, known as folkpsychology, naïve psychology, or theory of mind. The central idea is that people, and perhaps other animated objects, are intentional agents who act, and cause others to act, on the basis of internal motivations. ${ }^{k}$

6 Bzw. Ahnen, die in irgendeiner Form immer noch unter den Lebenden präsent sind.

7 Atran 2004: 57.

8 Atran 2004: 59. 
Das System ist hochsensibel und „entdeckt“ auch hinter Vorgängen, die nicht auf Handlungen einer Person zurückgehen, einen intentional handelnden Verursacher, z.B. wird eine Bewegung in einem Gebüsch auf ein Tier und nicht auf den Wind zurückgeführt.

Das entsprechende Modul im Gehirn wird insbesondere in einer neuen Umgebung, oder unter Bedingungen, in denen der Wahrnehmende auf irgendeine Weise verwundbar ist, besonders leicht einen Verursacher entdecken. Es könnte sich ja um eine Gefahr handeln. Auf diese Art erscheinen z.B. Kindern nächtliche Schatten im Schlafzimmer als bedrohliche Gestalten.

Atran geht davon aus, dass die Vorstellung übernatürlicher Verursacher auf diesem Mechanismus beruht: Vorkommnisse, die nicht auf Anhieb durch andere Gründe erklärt werden können, erwecken im Gehirn den Eindruck, ein nicht-sichtbarer Handelnder sei dafür verantwortlich.

Wenn diese nicht-sichtbaren Handelnden mit den Verstorbenen in Zusammenhang gebracht werden, die nach ihrem Tod ebenfalls nicht-sichtbar sind, früher aber durchaus Handelnde waren, entsteht ein Erklärungsmuster für den Toten- bzw. Ahnengeist: Die Toten sind in unsichtbarer Form präsent und können immer noch Einfluss auf die Lebenden und deren Umgebung nehmen.

Die entsprechende Vorstellung wird irgendwann kulturell integriert und zu einem religiösen Inhalt umgestaltet. Atran schreibt:

Supernatural agency is the most culturally recurrent, cognitively relevant, and evolutionary compelling concept in religion. The concept of the supernatural agent is culturally derived from innate cognitive schema, 'mental modules,' for the recognition and interpretation of agents, such as people and animals. By 'culturally derived,' I mean that numbers of people acting together casually manipulate innate, specieswide processing schema in historically contingent ways, much as makeup and masks involve culturally collective, contingent, causal manipulation of innate, modular sensibilities to secondary sexual characteristics and human facial cues. ${ }^{9}$

Der Umstand, dass so gut wie jede menschliche Kultur eine solche Vorstellung besitzt, spricht für den Ursprung des Geisterglaubens im gleichartigen Aufbau des Gehirns des Menschen.

\section{Ahnenkult als religiöser Akt in verschiedenen Kulturen}

Dieser gefühlte Einfluss der verstorbenen Ahnen erklärt auch das häufige Vorhandensein von Ahnen- bzw. Totenkulten in den meisten menschlichen Gesellschaften: Die Verstorbenen werden in irgend einer Weise als immer noch anwesend gedacht. Die Lebenden versuchen im Rahmen festgelegter Formen und Rituale, mit diesen Toten in Kontakt zu treten oder ihre Handlungen zu beeinflussen.

Der Hinduismus enthält schon seit der vedischen Zeit solche Rituale, die sich mit dem Sterben, dem Tod und der Versorgung der Ahnen nach dem Tod befassen. Der Buddhismus lehnte zwar viele Konzepte der vedischen Religion ab, die alte Idee der geisterhaft präsenten Ahnen wurde jedoch übernommen. Mit dem Peta, wie er uns in den Geschichten des Petavatthu begegnet, nahmen die Totengeister eine eigene buddhis- 
tische Gestalt an: Hier ist der Peta eine gequälte Kreatur, die als abschreckendes Beispiel für die Gläubigen dient und sehr anschaulich die Auswirkungen falscher Lebensführung auf die Existenz nach dem Tod zeigt.

Die Geistervorstellung des mittelalterlichen Europa ist ebenfalls das Ergebnis eines längeren Prozesses der Inkorporation von vorchristlichem Gedankengut in einen christlich-religiösen Kontext: Die Römer kannten die Feste der Lemuria und der Parentalia, bei denen jeweils der (schädigenden-fremden) Totengeistern und der (wohlgesonnenen-vertrauten) verstorbenen Ahnen gedacht wurde. Zu diesem Zeitpunkt waren die Konzepte solcher Ahnen- und Totengeister also schon in das religiöse System integriert. Für die anderen vorchristlichen europäischen Kulturen kann von ähnlichen Praktiken ausgegangen werden.

Mit der Christianisierung Europas wurden Elemente der vorher bestehenden Religionen von der Kirche übernommenundteilweise umgedeutet. Der Gespensterglaube, der aufgrund der tiefen Verwurzelung im der menschlichen Psyche immer auf irgendeiner Ebene präsent ist, bildet davon keine Ausnahme.

Die kirchlichen Feiertage Allerheiligen und Allerseelen stellen ganz offensichtlich eine Aneignung dieses Themenkomplexes durch die Kirche dar. Die Popularisierung der Feste fällt mit der Entwicklung der kirchlichen Doktrin vom Fegefeuer zusammen. Das Fegefeuer als ein Zwischenort, an dem die Verstorbenen geläutert werden, bevor sie in den Himmel kommen, erlaubt es den Lebenden durch gute Werke (Messen, Stiftungen etc.), Einfluss auf den nachtodlichen Zustand der Verstorbenen zu nehmen in einem Rahmen, der durch die Kirche vorgegeben wird, und in dem ihre Vertreter als Mediatoren wirken.

\section{Die parallele Struktur der religiösen Gespenstergeschichte}

Die Handlungsabfolge einer typischen Begegnung ist in den Texten aus dem mittelalterlich-christlichen und dem südasiatischen Theravāda-buddhistischen Kulturkreis fast genau gleich, es lassen sich 4 bzw. 6 Phasen unterscheiden:

- Eine Vorgeschichte wird erzählt, die handelnden Personen werden vorgestellt.

1. Der Geist erscheint.

2. Der Geist wird als solcher erkannt, man fragt nach seinem jetzigen Zustand.

3. Der Geist erklärt sein Vergehen und seine Strafe.

4. Der Geist erklärt den Weg zur (eigenen) Erlösung.

- Die Lebenden ziehen Konsequenzen aus den Geschehnissen.

Dieses Grundschema gilt nicht nur für die beiden Geschichten, es lässt sich vielmehr auf eine ganze Reihe von Erzählungen aus den jeweiligen Traditionen anwenden. In beiden Fällen dienen die Geister als „Sprachrohr“ der jeweiligen religiösen Autoritäten und beziehen sich auf doktrinäre Grundsätze, die sie verletzt haben: die Nandā verhielt sich unethisch und büßt dafür mit einer Wiedergeburt in der Welt der Pretas; der bayerische Dienstmann (ministerialis) hat zwar gute Werke getan, allerdings aus eitler Ruhmsucht (vana gloria), nicht aus christlicher Nächstenliebe (caritas). Die Gespenster schildern jeweils, häufig sehr ausführlich, die Qualen, die sie als Strafe für ihre Verfehlungen erleiden müssen. An diesen Teil schließt sich dann oftmals noch eine Ermahnung an, die dazu dient, den Weg zu einer Erlösung von solchen Leiden zu weisen. Die Beschreibung 
ist manchmal ausführlich: Man soll z.B. ein bestimmtes Objekt spenden, oder eine bestimmte Handlung vollziehen, in anderen Fällen ergibt sie sich von selbst aus den Verfehlungen der Verdammten in der Geschichte. Ganz allgemein kann man davon ausgehen, dass die Interessen der Geister in solchen Geschichten sich mit denen der jeweiligen religiösen Autorität stark überschneiden.

\section{Religiösen Organisationsstrukturen und religiöse Inhalte}

Die beiden Texte entstanden jeweils in einer Zeit, die von religiösen Vorstellungen im Umbruch gekennzeichnet ist: In Indien bildete sich ab dem fünften vorchristlichen Jahrhundert ein Wanderasketenwesen aus, das sich in vielen Punkten von der brahmanischen religiösen Orthopraxie dieser Zeit abgrenzte. Der Buddhismus ist der bekannteste Vertreter dieser Strömung. Der buddhistische Orden war auf Spenden der Laienanhänger angewiesen und stand in direkter Konkurrenz zu den etablierten brahmanischen Priestern und dem gesamten brahmanischen Gedanken- und Ritualsystem.

Robert Decaroli argumentiert in seinem Buch Haunting the Buddha ${ }^{10}$, dass es eine besondere Stärke des frühen Buddhismus war, sich als Autorität für die verstorbenen Ahnen zu etablieren: Klostergründungen fanden z. B. in der Nähe von Gräberfeldern statt und die Anwesenheit lokaler Geister und Gottheiten wird in den Ordensregeln und anderen Texten wie selbstverständlich angenommen. Diese Rolle der buddhistischen Mönche als Autorität und Mediator zwischen Lebenden und Toten kommt auch in den Geschichten des Petavatthu immer wieder zum Tragen: Die Geister können nur dann eine gute Wiedergeburt erlangen, wenn man Spenden an den buddhistischen Orden gibt. Das Petavatthu kann daher als eine Sammlung von Beispielen für ethisches Verhalten der Gläubigen und für die Konsequenzen nicht-ethischen Handelns gesehen werden: Das Schicksal nach dem Tod wurde als Motivation für richtiges Handeln im Leben und als Anreiz für Spenden genutzt.

In Europa ergab sich im dreizehnten Jahrhundert ebenfalls eine Konstellation von verschiedenen Gruppen von religiösen Experten, die für ihr Fortbestehen auf die Ressourcen angewiesen waren, die von den Gläubigen erwirtschaftet wurden. Der „Konflikt“ fand aber innerhalb des Rahmens der katholischen Kirche als Dachorganisation statt. Die Etablierung von Bettelorden (Dominikaner, Franziskaner, Augustiner, Karmeliten etc.), die sich in vielen Punkten sowohl von der kirchlichen Verwaltungshierarchie als auch von den vorher existierenden monastischen Orden unterschieden, ist die Konsequenz eines lange schwelenden Konfliktes um das Verhältnis der Kirche zu weltlichen Gütern.

Diese Bettelorden waren wie die buddhistischen Mönche in Südasien ebenfalls auf Almosen angewiesen. Die eindrückliche Predigt vor den Laien, die auch Geschichten von friedlosen Geistern enthielt, war ein fester Bestandteil ihres Repertoires. Ronald Finucane schreibt:

Though the death-in-life theme was a commonplace in monastic circles, another source of such teachings - which was aimed at laymen - was the friars of the thirteenth century, especially Dominicans and Franciscans. Their sermons brought the message in unambivalent terms to the laity. These friars knew what they were doing, they knew how to teach through 
example, how to preach - when to tell a joke or a frightening story of tortured ghosts, when to call up fire and brimstone."

In diesem Zusammenhang ist auch dieGeschichte des bayerischen Dienstmannes zu sehen: ein abschreckendes Beispiel für falsches Verhalten, indirekt eine Handlungsanweisung für richtiges Verhalten und rechtgläubige Gesinnung.

\section{Parallelen und Divergenzen}

Die religiösen Experten der beiden Religionen Christentum und Buddhismus beanspruchten für sich eine Mittlerrolle zwischen Mensch und dem Übernatürlichen, auch im Bereich der nachtodlichen Existenz.

Im Austausch für ihre spirituellen Dienste wurden beide Gruppen durch Spenden aus der Laienschaft versorgt. Eine Möglichkeit, diese Spenden besonders reichlich fließen zu lassen, war der Hinweis auf die nachtodliche Existenz, die direkt oder indirekt mit der Spendenbereitschaft verknüpft wurde. Sowohl Christentum als auch Buddhismus beschäftigen sich ausgiebig mit dem Themenkomplex des Todes und der Toten. Daher war es naheliegend, dass diese „Kernkompetenz“ der religiösen Experten der Laienschaft besonders deutlich gemacht wurde. Der buddhistische Orden betonte den Verdienst ( $p u \tilde{n} \tilde{n} a$ ), der durch das Spenden angehäuft werden konnte, im Christentum findet sich ab dem elften nachchristlichen Jahrhundert die Vorstellung des Fegefeuers (eine ,eschatological revolution"'12), in dem jeder, der nicht völlig frei von Sünden ist, eine bestimmte Zeit verbringen muss, bevor er in den Himmel kommt. Die Bettelorden propagieren ein Verfahren, in dem der Verstorbene ,in einem individuellen, vor einem Notar aufgesetzten Testament bestimmte Geldsummen [hinterlässt], mit denen in möglichst vielen Kirchen eine möglichst große Zahl von Seelenmessen gekauft werden soll, um seine endgültige Erlösung aus dem Fegefeuer zu beschleunigen." 13

Der entsprechende Austausch materieller gegen spirituelle Güter ist zwar theologisch völlig unterschiedlich begründet und konstruiert, läuft in der Praxis jedoch genau parallel ab.

In beiden Religionen gab es also eine Reihe historischer Entwicklungen die ähnlich verliefen, wodurch letztendlich dieses gleichartige Ergebnis erzielt wird:

1. Die Kultur kennt die Vorstellung einer nachtodliche Existenz.

2. In der Religion wird die Vorstellung vom Einfluss des Individuums auf die eigene nachtodliche Existenz propagiert, d. h. es greift ein System von Ursache und Wirkung (Handlungen im Leben wirken sich auf das eigene Schicksal nach dem Tod aus).

3. In der Religion nimmt eine Gruppe von religiösen Experten eine besondere Vermittlerrolle zwischen Menschen und dem Übernatürlichen wahr.

4. Die Gruppe der religiösen Experten finanziert sich aus Spenden der Laienschaft

Die Vorstellung von übernatürlichen Wesenheiten und einem Nachleben als Geist wird durch die Funktionsweise des Gehirns begünstigt. Religionen können an diesem Substrat ansetzen und solche Geistervorstellungen in ihre eigene Theologie integrieren. 
Unter den richtigen Umständen fällt den religiösen Experten einer Religion die Rolle eines Mittlers zwischen den Gläubigen und ihren verstorbenen Angehörigen zu. Sowohl der frühe indische Buddhismus als auch das mittelalterliche Christentum haben ein solches Austauschsystem zwischen Lebenden und Verstorbenen entwickelt. Die strukturellen Übereinstimmungen zwischen den Gespenstergeschichten des Petavatthu und des Dialogus Miraculorum sind ein literarischer Beleg für diese parallele Entwicklung.

\section{Literaturverzeichnis}

Atran, Scott. 2004 [2002]. In Gods We Trust - The Evolutionary Landscape of Religion. Oxford / New York, u. a., Oxford University Press.

Decaroli, Robert. 2004. Haunting the Buddha - Indian Popular Religions and the Formation of Buddhism. Buffalo / New York, u. a. Oxford University Press.

Finucane, Ronald C. 1984. Appearances of the Dead -A Cultural History of Ghosts. Buffalo. New York, Prometheus Books.

Herles, Helmut. 1990. Von Geheimnissen und Wundern des Caesarius von Heisterbach. Bonn, Bonnvier Verlag.

Rhys Davids, C.A.F. (Hrsg.). 1942. The Minor Anthologies of the Pali Canon, Part IV. Vimanna Vatthu: Stories of the Mansions and Peta Vatthu: Stories of the Departed. Translated by Jean Kennedy and Henry S. Gehman. London, Pali Text Society.

Schmitt, Jean-Claude. 1995 (franz. Originalausgabe 1994). Die Wiederkehr der Toten Geistergeschichten im Mittelalter. Stuttgart, Klett-Cotta.

Stede, Wilhelm. 1914. Die Gespenstergeschichten des Petavatthu. Leipzig, Harrassowitz. 